\title{
physioswiss résilie sa convention tarifaire avec santésuisse
}

Les physiothérapeutes indépendants voient leur rémunération stagner depuis douze ans. Toutes les négociations avec santésuisse portant sur une augmentation de la valeur du point ont échoué. physioswiss, I'Association suisse de physiothérapie, tire les conséquences qui s'imposent pour la branche et résilie la convention tarifaire au 31 décembre 2009.

Christian Mehr

Secrétaire générale

Correspondance:

physioswiss

Stadthof

Centralstrasse $8 \mathrm{~b}$

CH-6210 Sursee

Tél. 0419266969

Fax 0419266999

info@physioswiss.ch

www.physioswiss.ch
Il était temps d'agir. En Suisse, les physiothérapeutes indépendants travaillent depuis douze ans au même prix, sur la base de la convention tarifaire de 1998. Ils génèrent un revenu brut moyen de CHF 89.- par heure. Ce montant doit couvrir les frais de loyer, de matériel et d'installation. Comme aucune adaptation au renchérissement n'a été effectuée depuis l'entrée en vigueur de la convention, le salaire réel des physiothérapeutes décroît depuis des années et a atteint un seuil critique.

Depuis trois ans, physioswiss a négocié intensivement une augmentation de la valeur du point avec santésuisse . Les entretiens ont été âpres et santésuisse ne se montrait pas prête à faire le moindre compromis sur la structure tarifaire ou la base des calculs. Pour ne pas compromettre l'avenir de la profession - dans ces conditions financières, bientôt personne ne voudra plus embrasser la profession de physiothérapeute - physioswiss a tiré les conséquences et dénonce la convention tarifaire qui la lie à santésuisse fin décembre 2009.

Par cette résiliation, physioswiss adopte une position claire envers santésuisse: physioswiss revendique un salaire équitable pour le travail de haute qualité fourni par ses membres. Ceci est l'objectif principal des négociations tarifaires qu'il s'agira d'entamer sans délai au début de l'année prochaine avec santésuisse.

Quelles sont les conséquences de cette résiliation pour les médecins - peuvent-ils continuer à prescrire de la physiothérapie?

La résiliation de la convention tarifaire entre physioswiss et santésuisse n'a aucune conséquence sur vous en tant que médecin. Vous pouvez continuer de prescrire de la physiothérapie comme d'habitude. Dans le cas improbable où d'autres modifications surviendraient, physioswiss, l'Association suisse de physiothérapie, vous informera en temps voulu.

\section{La physiothérapie prescrite par les médecins sera-t-elle toujours prise en charge par} l'assurance de base?

Oui, les coûts seront comme jusqu'à présent pris en charge par l'assurance de base. La facture passe comme auparavant par le tiers payant, soit directement des physiothérapeutes vers les assurances.

Y-a-t-il des conséquences pour les patients?

Si vos patients ont des questions sur la résiliation de la convention entre physioswiss et santésuisse, vous pouvez leur dire que ceci n'a aucune conséquence pour eux. La physiothérapie va continuer d'être prescrite par les médecins et sera prise en charge par l'assurance de base. La facture passera comme auparavant par le tiers payant, directement des physiothérapeutes vers les assurances. Les patients ne doivent donc craindre ni dépenses administratives, ni de réduction du nombre de leurs séances de physiothérapie ou de refus de pouvoir en bénéficier.

Comme l'exige la convention tarifaire, physioswiss et santésuisse devront reprendre les négociations tarifaires sans tarder. physioswiss a pour objectif de négocier un salaire équitable pour le travail de grande qualité fourni par ses membres.

Si vous avez d'autres questions sur la résiliation de la convention tarifaire, vous pouvez nous contacter surinfo@physioswiss.ch ou au 0419266969.

L'association professionnelle physioswiss et ses membres sont ravis de poursuivre une bonne collaboration avec vous, chers collègues médecins. 